\title{
Simulation of the Carton Erection for the Rubber Glove Packing Machine Using Finite Element Method
}

\author{
Kawin Jewsuwun and Chakrit Suvanjumrat \\ ${ }^{1}$ Department of Mechanical Engineering, Faculty of Engineering, Mahidol University, Salaya, Nakorn Pathom, 73170, Thailand \\ ${ }^{2}$ Laboratory of Computer Mechanics for Design (LCMD), Department of Mechanical Engineering, Faculty of Engineering, Mahidol University, \\ Salaya, Nakorn Pathom, 73170, Thailand
}

\begin{abstract}
The rubber glove packing machine had been designed an important function which worked with folding carton. Each folded paper carton would be pulled to be erected by vacuum cups. Some carton could not completely form because of an unsuitable design of the erector. Cartons were collapsed or buckling while pulled by vacuum cups that cause to sudden stop the packing process and affect to number and cost of rubber glove production. This research aimed to use simulation method to erect the folded carton. Finite element (FE) model of the rubber glove carton was created with shell elements. The orthotropic material properties were employed to specify FE model for analysis erection behavior of the folding carton. Vacuum cups number, positions and rotation points were simulated until obtained a good situation of the folding carton erector. Subsequently, finite element analysis results will be used to fabricate erector of the rubber glove packing machine in a further work.
\end{abstract}

\section{Introduction}

The competition to increase productivity has effected to the rubber glove industry. The production line of rubber gloves is divided into the rubber glove production and packaging. The packaging process required a lot of manpower starting from removing rubber gloves from formers, counting of gloves, folding of cartons and packaging of gloves into carton. Currently, the packaging machine becomes another alternative to replace workers and effectively reduce the process time and cost. Unfortunately, the packaging machine is encountered several issues during carton erection process. The important problem may be buckling of the carton during the transition between flattened and erected states.

The carton which is used in glove industry generally, made from paperboard. The crease on carton board happens by pressing die which makes a groove line on one side and a protuberant line on the other side. Recently, several works have been performed to study the material behavior of paperboard. Carlsson et al. [1] investigated crease stiffness and bending strength of crease zones. A parallel beam model was used to describe bending behavior of locally delaminated zones, which were creased zones of paperboard. Nagasawa et al. [2] investigated creasing characteristic by varying effect of crease depth and crease deviation. The 3-D analytical estimation and 2-D finite element method consisted of carton panel and the crease springs have been developed to study the stiffness characteristic of creases and panels of a carton. The resistive moment resulting from carton crease and force required for folding cartons was investigated [3-6]. Recently, the carton manufacturers have forced to use lighter-weighted carton in order to reduce wasted and production cost. This packaging legislation had decreased carton stiffness which may lead to be failure of the folding process. In order to tackle these problems, the FE model of carton processing is developed to simulate the behavior of folded carton during the complex packing operations. The orthotropic constitutive model was used to model nonlinear behavior of carton wall. The linear elastic model is insufficient since stress of the carton wall in thickness directions being low mechanical resistant and tend to cause buckling of the individual plies and may result in unacceptable damage of the inter-laminar bonds [7].

This research aimed to develop finite element model of carton unfolding process using packaging machine. The orthotropic constitutive model is then used to describe carton behavior, while the multiple point constraint is applied to model simplified crease between each panel. The model is then analyzed to observe important characteristic of folding cartons such as carton deformation and mechanical response.

\section{Finite element method}

The carton is composed of four rectangular panels with $\mathrm{L} \times \mathrm{W} \times \mathrm{H}$ of $220 \times 120 \times 70 \mathrm{~mm}$. The erector arm has $\mathrm{L}-$ shape with a rotating point to rotate up and down. The height of the erector arm (B) can be adjusted regarding to the installation position. The length of erector arm for 
installation vacuum cup is $232 \mathrm{~mm}$. The vacuum cups which is a diameter of $19.2 \mathrm{~mm}$, are installed on the erector arms. The number of vacuum cups can be selected to install on the erector arm. Fig. 1 shows schematic of the carton and the erector arm that bring to analyze with finite element method (FEM). Fig. 2 shows FE model to simulate the carton erection by using commercial finite element software, MSC. Patran. The quadratic shell elements are used to create skillet model. The skillet is simplified by modeling with only four panels. The FE model of vacuum cups is also used the quadratic shell elements. The erector arms are used the tetrahedron elements. Description of elements which used to create FE model show in Table 1.

Table 1. Types and amount of elements using for the FE model.

\begin{tabular}{|l|c|c|c|}
\hline \multicolumn{1}{|c|}{ Part } & Element type & Dimension & Amount \\
\hline Carton & Quadratic & 2-D & 1,219 \\
\hline Vacuum cup & Quadratic & 2-D & 111 \\
\hline $\begin{array}{l}\text { Elector arm I } \\
(\mathrm{B}=52.5, \mathrm{C}=30.0 \\
\mathrm{mm})\end{array}$ & Tetrahedron & 3-D & 1,046 \\
\hline $\begin{array}{l}\text { Elector arm II (B }= \\
52.5, \mathrm{C}=40.0 \mathrm{~mm})\end{array}$ & Tetrahedron & 3-D & 1,180 \\
\hline $\begin{array}{l}\text { Elector arm III (B }= \\
67.5, \mathrm{C}=30.0 \mathrm{~mm})\end{array}$ & Tetrahedron & 3-D & 1,092 \\
\hline $\begin{array}{l}\text { Elector arm IV (B }= \\
70.0, \mathrm{C}=40.0 \mathrm{~mm})\end{array}$ & Tetrahedron & 3-D & 1,185 \\
\hline
\end{tabular}

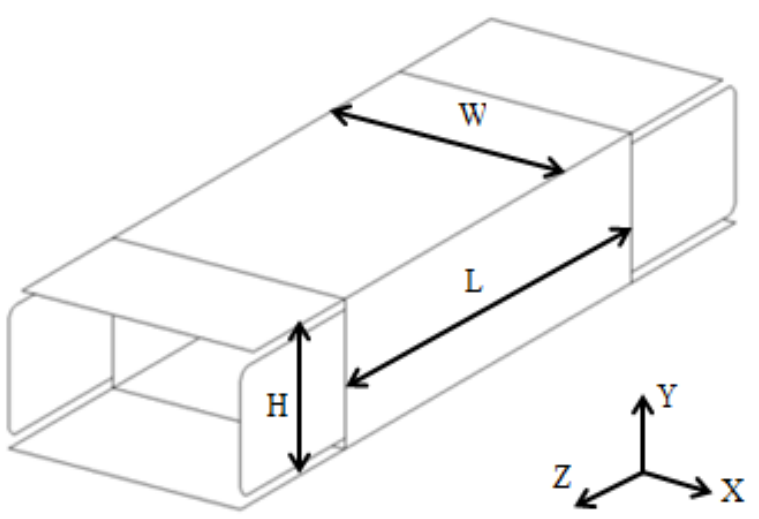

(a)

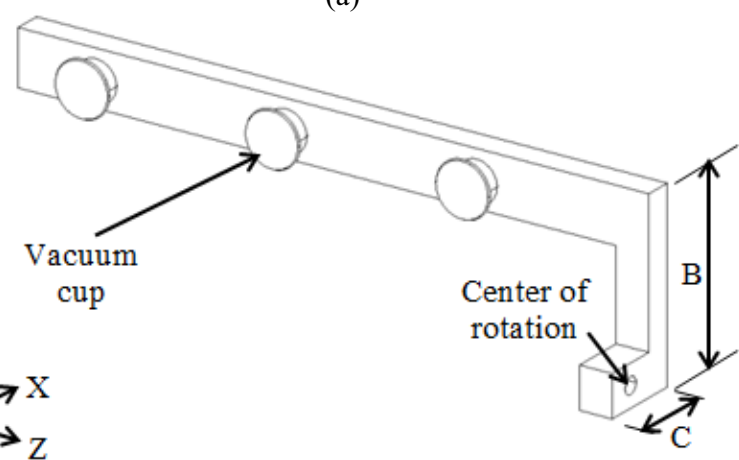

(b)

Figure 1. Schemetric of: (a) a carton and (b) an elector arm

Boundary conditions of the FE model of carton erection were defined to analyze deformation of cartons with different positions of erector arms and number of vacuum cups. Multiple point constraint (MPC) was used to model the crease and connect each panels of a carton together. The fixed constraint was applied to simulate the attached vacuum cups under the bottom of the skillet as shown in Fig. 2c.

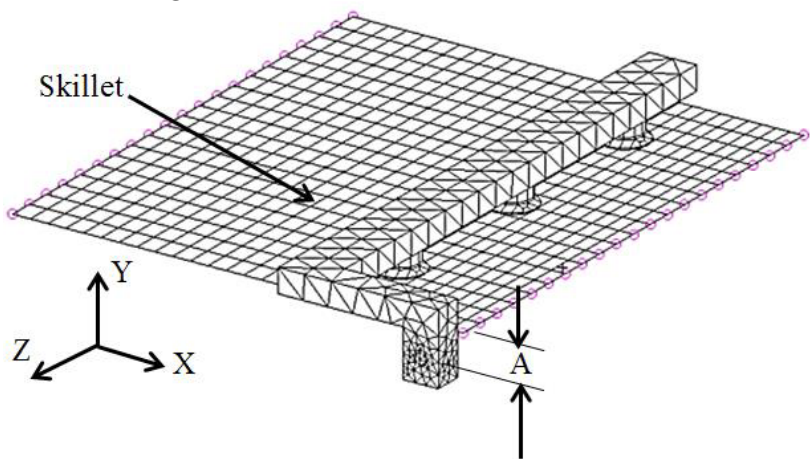

(a)

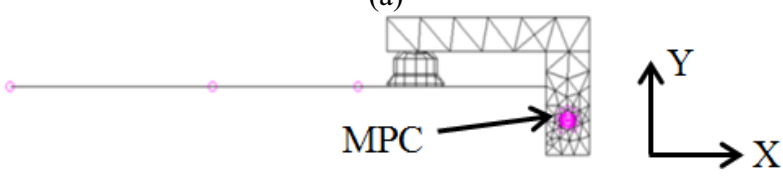

(b)

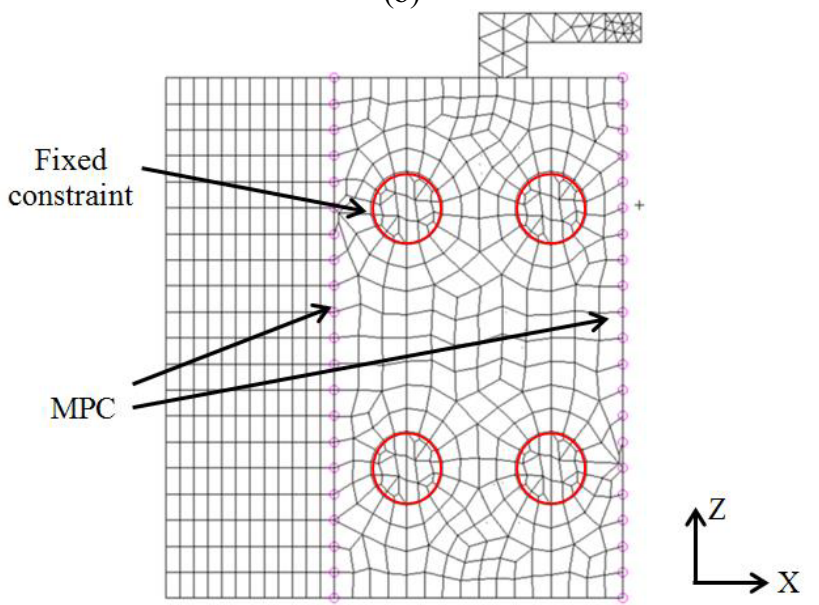

(c)

Figure 2. The FE model of the carton erection in: (a) isomatric view, (b) front view and (c) bottom view.

The MPC was also used to model a center of rotation of the erector arm. The vacuum cups were assigned to contact with skillet element. The contact conditions were applied to be "glued after contact" to simulate suction of the vacuum cups. The FE model of a skillet, vacuum cups and erector arm is assembled in the different position. Fig. 3 shows FE models which are created to analyze a suitable position of the carton erections. The center of rotation of erection arm I and III was on the level of skillet while the center of rotation of erection arm II and IV was lower than skillet of $10 \mathrm{~mm}$. The FE model of carton erections was analyzed by using the FE software, MSC.Nastran. Six conditions of the carton erection were simulated in this research.

The orthotropic constitutive model has been used to describe the nonlinear behavior of carton wall during unfolding process. The carton board can be considered orthotropic since most of its fibers have been reoriented during manufacturing, resulted in different mechanical strength between oriented direction and the others. The oriented direction is commonly known as machine 
direction (MD), while the perpendicular direction is known as cross direction (CD). The $\mathrm{MD}$ and $\mathrm{CD}$ direction are laid on the carton panels. In addition, the mechanical properties in the thickness direction are also different from the other two directions due to laminar construction, this thickness direction may be abbreviated as ZD. The element thickness of the vacuum cup was 1 $\mathrm{mm}$ while carton board was $0.8 \mathrm{~mm}$. The orthotropic material model can be written in the form of compliance matrix as:

$\left\{\begin{array}{l}\varepsilon_{11} \\ \varepsilon_{22} \\ \varepsilon_{33} \\ \gamma_{12} \\ \gamma_{13} \\ \gamma_{23}\end{array}\right\}=\left[\begin{array}{cccccc}\frac{1}{E_{1}} & \frac{-v_{21}}{E_{2}} & \frac{-v_{31}}{E_{3}} & 0 & 0 & 0 \\ \frac{-v_{12}}{E_{1}} & \frac{1}{E_{2}} & \frac{-v_{32}}{E_{3}} & 0 & 0 & 0 \\ \frac{-v_{13}}{E_{1}} & \frac{-v_{23}}{E_{2}} & \frac{1}{E_{3}} & 0 & 0 & 0 \\ 0 & 0 & 0 & \frac{1}{G_{12}} & 0 & 0 \\ 0 & 0 & 0 & 0 & \frac{1}{G_{13}} & 0 \\ 0 & 0 & 0 & 0 & 0 & \frac{1}{G_{23}}\end{array}\right]\left\{\begin{array}{c}\sigma_{11} \\ \sigma_{22} \\ \sigma_{33} \\ \sigma_{12} \\ \sigma_{13} \\ \sigma_{23}\end{array}\right\}$

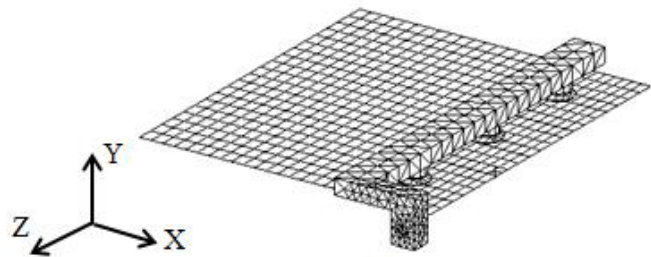

(a)

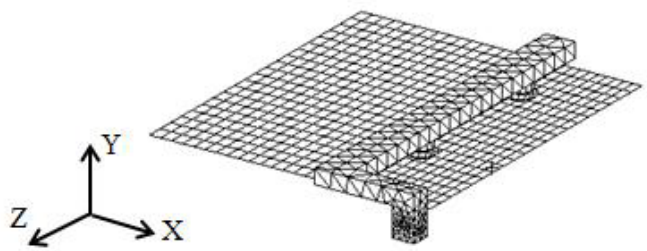

(c)

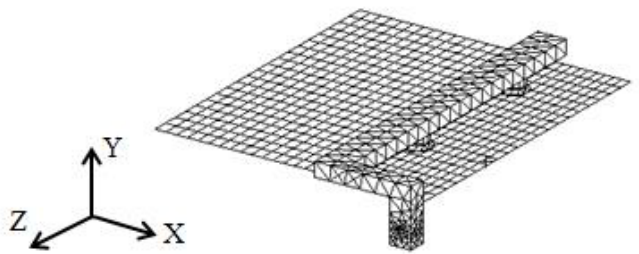

(e) where $E_{i j}, G_{i j}$ and vij is Young's modulus, shear stress and Poisson's ratio in $\mathrm{i}$ and $\mathrm{j}$ direction, respectively. The first direction of Young's modulus $\left(E_{l}\right)$ which is corresponding to MD was aligned to the principle $\mathrm{x}$-axis of the model while the second direction $\left(E_{2}\right)$ is corresponding to $\mathrm{CD}$ in $\mathrm{z}$ direction. The third direction $\left(E_{3}\right)$ was normal to shell elements. The mechanical properties of orthotropic material which are obtained from [8] shown in Table 2.

Table 2. Mechanical properties of the caton board.

\begin{tabular}{|c|c|}
\hline Property & Value \\
\hline $\mathrm{E}_{1}(\mathrm{MPa})$ & 4460 \\
\hline $\mathrm{E}_{2}(\mathrm{MPa})$ & 2385 \\
\hline $\mathrm{E}_{3}(\mathrm{MPa})$ & 22 \\
\hline $\mathrm{G}_{12}(\mathrm{MPa})$ & 1272 \\
\hline $\mathrm{G}_{13}(\mathrm{MPa})$ & 81 \\
\hline $\mathrm{G}_{23}(\mathrm{MPa})$ & 68 \\
\hline$v_{12}$ & 0.15 \\
\hline$v_{13}$ & 0.08 \\
\hline$v_{23}$ & 0.21 \\
\hline$\rho\left(\mathrm{kg} / \mathrm{m}^{3}\right)$ & 615 \\
\hline
\end{tabular}

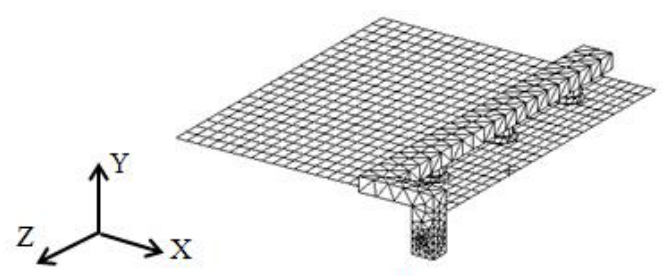

(b)

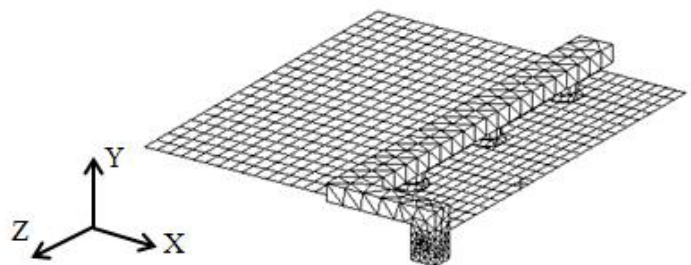

(d)

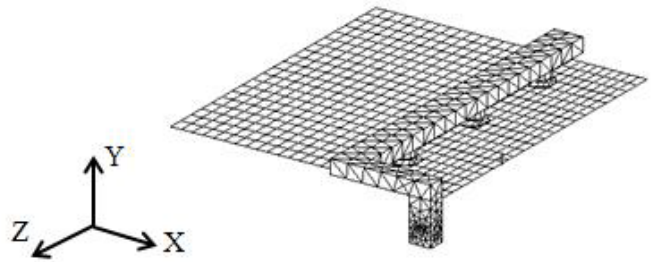

(f)

Figure 3. The FE models of the carton erections by variation of: (a) an elector arm I with three vacuum cups, (b) an erector arm II with three vacuum cups, (c) an elector arm III with two vacuum cups, (d) an erector arm III with three vacuum cups, (e) an elector arm IV with two vacuum cups and (f) an erector arm IV with three vacuum cups.

\section{Results and discussion}

The finite element analysis results of carton erection process with different erector arm positions are shown in Fig. 4. The stress and deformation of erected carton which assembled with an erector arm were illustrated. The red portion of the model indicated maximum stress, while the minimum was indicated by blue color of the contour. The erector arm I and II with three vacuum cups (Fig. 4a and 4b) and an erector arm III, IV with two vacuum cups (Fig $4 \mathrm{c}$ and $4 \mathrm{e}$ ) is shown carton erection at the angle of $45^{\circ}$ from the initial skillet position. The erected carton with an erector arm III, IV with three vacuum cups (Fig. $4 \mathrm{~d}$ and $4 \mathrm{f}$ ) is shown erection at the angle of $90^{\circ}$. The comparison between analyzed results of the different erector arm models shows the carton model with an erector arm I and II yield more buckling than an 
erector arm III and IV. The over buckling effect may lead to warpage of carton board near the crease corner, which may be divergence of the simulation results. Two vacuum cups which installed on the erector arm III forced bending of the carton panel. The large concentrated stress happened on the panel by two vacuum cups were more

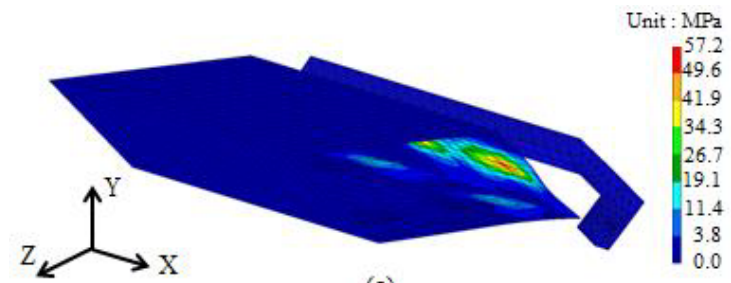

(a)

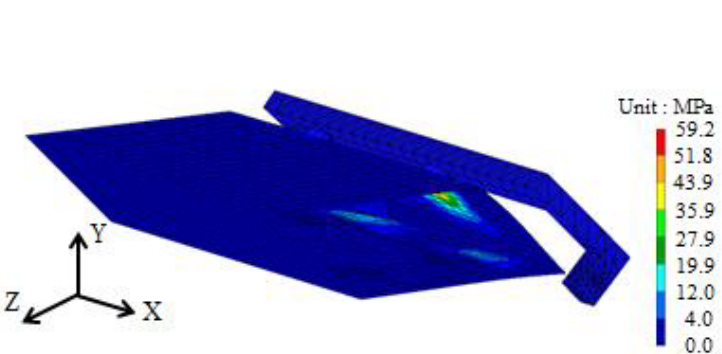

(c)

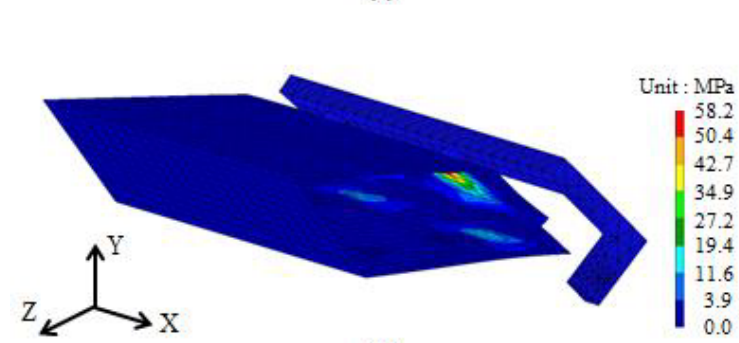

(e) than three vacuum cups on the erector arm. The erector arm IV did not obtain the divergent result but it made carton panel delaminate. The advantage of 3-D orthotropic material is distinct wrinkle of the panel. Consequently, the erector arm III is appropriate to unfold this carton.

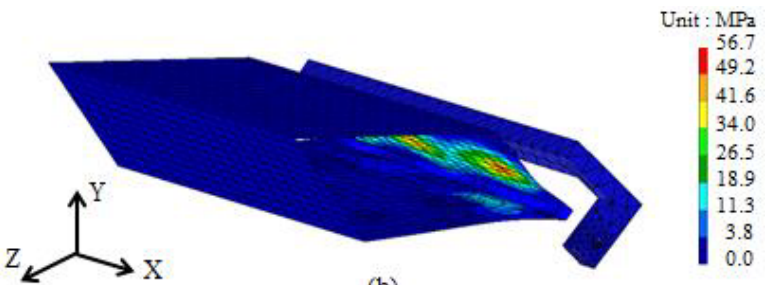

(b)

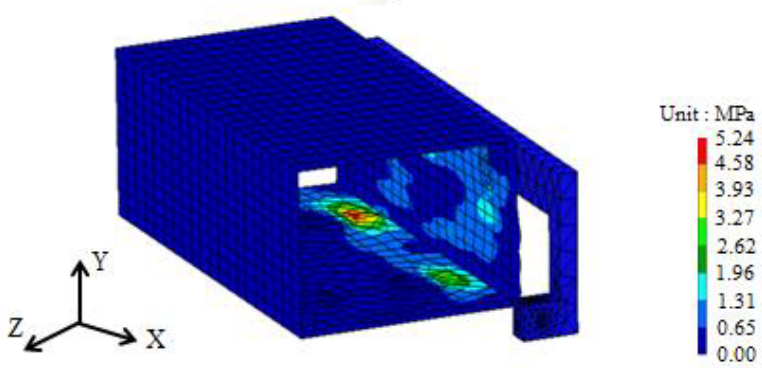

(d)

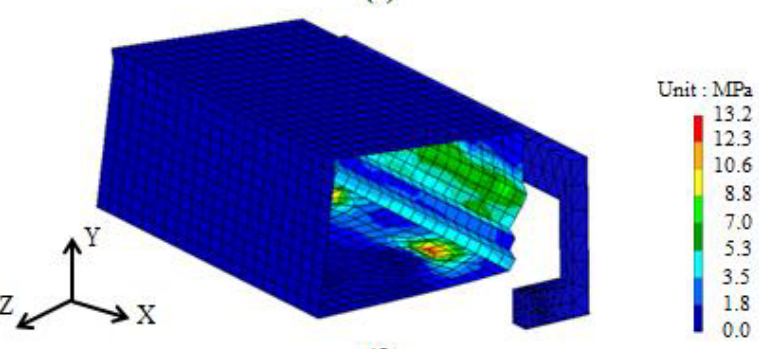

(f)

Figure 4. The FE simulation results of the carton erections by variation of: (a) an elector arm I with three vacuum cups, (b) an erector arm II with three vacuum cups, (c) an elector arm III with two vacuum cups, (d) an erector arm III with three vacuum cups, (e) an elector arm IV with two vacuum cups and (f) an erector arm IV with three vacuum cups.

\section{Conclusions}

The finite element models of carton erection process were developed. The model consisted of skillet, erector arms and vacuum cups. The skillet and vacuum cups were modeled with 2-D shell elements while erector arm was modeled with 3-D solid elements. The skillet was simplified by modeling with four panels. The orthotropic material model was used to describe the nonlinear behavior of cartons, while the erector arm was defined as linear isotropic material. The vacuum cups were assigned to glued contact with the skillet and the erector arm was rotated $90^{\circ}$ from initial skillet position. The finite element models of six different carton erections were analyzed to study the effects of machine design. Buckling and distortion effects occurred on carton wall during erection process could be also predicted by FE results. The FE models could be used to study for appropriate geometry and position of the carton erection mechanism and give useful information and understanding for designing and development of the glove packaging machine in the near future.

\section{References}

1. L. Carlsson, A.D. Ruvo, C. Fellers, J. Mater. Sci. 18 (1983)

2. S. Nagasawa, Y. Fukuzawa, T. Yamaguchi, S. Tsukatani, I. Katayama, J. Mater. Process. Tech. 140 (2003)

3. F. Cannella, J.S. Dai, J. Mech. Eng. Sci. 220 (2006)

4. A. Giampieri, U. Perego, R. Borsari, Int. J. Solids Struct. 48 (2011)

5. L. Mentrasti, F. Cannella, M. Pubilli, J.S. Dai, Int. J. Solids Struct. 50 (2013)

6. H. Huang, A. Hagman, M. Nygards, Mech. Mater. 69 (2014)

7. D.M. Sirkett, B.J. Hicks, C. Berry, G. Mullineux, A.J. Medland, J. Mech. Eng. Sci. 220 (2006)

8. D.M. Sirkett, B.J. Hicks, C. Berry, G. Mullineux, A.J. Medland, J. Mech. Eng. Sci. 221 (2007). 